\title{
Parity effect and spontaneous currents in superconducting nanorings
}

\author{
Sergei V. Sharov ${ }^{\mathrm{a}, \mathrm{b}}$ and Andrei D. Zaikin ${ }^{\mathrm{c}, \mathrm{d}, 1}$ \\ anstitut für Theoretische Festkörperphysik, Universität Karlsruhe, 76128 Karlsruhe, Germany \\ ${ }^{\mathrm{b}}$ Institute for Physics of Microstructures, Russian Academy of Sciences, 603950 Nizhny Novgorod, Russia, \\ ${ }^{\mathrm{c}}$ Forschungszentrum Karlsruhe, Institut für Nanotechnologie, 76021 Karlsruhe, Germany, \\ ${ }^{\mathrm{d}}$ I.E.Tamm Department of Theoretical Physics, P.N.Lebedev Physics Institute, 119991 Moscow, Russia
}

\begin{abstract}
New physical effects emerge from an interplay between the electron parity number and persistent currents in superconducting nanorings. An odd electron, being added to the ring, produces a countercurrent which may substantially modify the ground state properties of the system. In superconducting nanorings with an embedded normal metal layer a novel " $\pi / N$-junction" state can occur for the odd number of electrons. Changing this number from even to odd yields spontaneous supercurrent in the ground state of such rings without any externally applied magnetic flux. Further peculiar features of the parity effect are expected in structures with resonant electron transport across the weak link.
\end{abstract}

Key words: superconductivity, persistent current, parity effect, nanorings, $\pi$-junctions PACS: 74.78.Na, 73.23.Ra, 74.45.+c, 74.50.+r

\section{Introduction}

It is well established both theoretically and experimentally $[1,2,3,4,5,6]$ that thermodynamic properties of isolated superconducting systems are sensitive to the parity of the total number of electrons $\mathcal{N}$ despite this number may be macroscopically large. The fundamental difference between superconductors with even and odd $\mathcal{N}$ is due to the fact that the number of electrons in the condensate of Cooper pairs should necessarily be even. Hence, for odd $\mathcal{N}$ at least one electron remains unpaired having an extra energy equal to the

\footnotetext{
1 Corresponding author. E-mail: zaikin@int.fzk.de
}

superconducting gap $\Delta$. This difference - hindered by the entropy factor at sufficiently high temperatures becomes important in the low temperature limit.

Can the supercurrent be affected by this parity effect? Recently we have argued [7] that (a) the answer to this question is positive and (b) in superconducting nanorings interrupted by a weak link parity-restricted supercurrent can strongly deviate from one evaluated for the grand canonical ensemble. In this paper we will extend our previous analysis focusing it on superconducting rings with embedded piece of a normal metal ( $S N S$ rings). We will consider transparent nanojunctions as well as structures with low transmission of $N S$ interfaces. In the latter case resonant effects become 
important leading to substantial modifications of the physical picture of the parity effect [7].

\section{Parity projection formalism}

In the subsequent analysis of parity-restricted persistent currents (PC) in superconducting nanorings we will employ the parity projection formalism $[4,5,6]$. Recapitulating the key points of this approach we will closely follow Ref. [5]. We will then derive a general formula for the circulating supercurrent in isolated superconducting rings with even/odd number of electrons.

The grand canonical partition function $\mathcal{Z}(T, \mu)=$ $\operatorname{Tr} e^{-\beta(\mathcal{H}-\mu \mathcal{N})}$ is linked to the canonical one $Z(T, \mathcal{N})$ as

$$
\mathcal{Z}(T, \mu)=\sum_{\mathcal{N}=0}^{\infty} Z(T, \mathcal{N}) \exp \left(\frac{\mu \mathcal{N}}{T}\right) .
$$

Here and below $\mathcal{H}$ is the system Hamiltonian and $\beta \equiv$ $1 / T$. Inverting this relation and defining the canonical partition functions $Z_{e}$ and $Z_{o}$ respectively for even $\left(\mathcal{N} \equiv \mathcal{N}_{e}\right)$ and odd $\left(\mathcal{N} \equiv \mathcal{N}_{o}\right)$ ensembles, one gets

$$
Z_{e / o}(T)=\frac{1}{2 \pi} \int_{-\pi}^{\pi} d u e^{-i \mathcal{N}_{e / o} u} \mathcal{Z}_{e / o}(T, i T u),
$$

where

$$
\begin{gathered}
\mathcal{Z}_{e / o}(T, \mu)=\frac{1}{2} \operatorname{Tr}\left\{\left[1 \pm(-1)^{\mathcal{N}}\right] e^{-\beta(\mathcal{H}-\mu \mathcal{N})}\right\} \\
=\frac{1}{2}(\mathcal{Z}(T, \mu) \pm \mathcal{Z}(T, \mu+i \pi T))
\end{gathered}
$$

are the parity projected grand canonical partition functions. For $\mathcal{N} \gg 1$ it is sufficient to evaluate the integral in (2) within the saddle point approximation

$$
Z_{e / o}(T) \sim e^{-\beta\left(\Omega_{e / o}-\mu_{e / o} \mathcal{N}_{e / o}\right)},
$$

where

$$
\Omega_{e / o}=-T \ln \mathcal{Z}_{e / o}(T, \mu)
$$

are the parity projected thermodynamic potentials,

$\Omega_{e / o}=\Omega_{f}-T \ln \left[\frac{1}{2}\left(1 \pm e^{-\beta\left(\Omega_{b}-\Omega_{f}\right)}\right)\right]$

and

$$
\Omega_{f / b}=-T \ln \left[\operatorname{Tr}\left\{( \pm 1)^{\mathcal{N}} e^{-\beta(\mathcal{H}-\mu \mathcal{N})}\right\}\right] .
$$

"Chemical potentials" $\mu_{e / o}$ are defined by the saddle point condition

$$
\mathcal{N}_{e / o}=-\partial \Omega_{e / o}\left(T, \mu_{e / o}\right) / \partial \mu_{e / o} .
$$

Let us emphasize that $\Omega_{f}$ is just the standard grand canonical thermodynamic potential and $\Omega_{b}$ represents the corresponding potential linked to the auxiliary partition function $\mathcal{Z}(T, \mu+i \pi T)$. This function can be conveniently evaluated by finding the true grand canonical partition function $\mathcal{Z}(T, \mu)$, expressing the result as a sum over the Fermi Matsubara frequencies $\omega_{f}=$ $2 \pi T(l+1 / 2)$ and then substituting the Bose Matsubara frequencies $\omega_{b}=2 \pi T l(l=0, \pm 1, \ldots)$ instead of $\omega_{f}$.

Consider now an isolated superconducting ring pierced by the magnetic flux $\Phi$. Making use of the above expressions one one can easily determine the equilibrium current $I_{e / o}$ circulating inside the ring:

$$
I_{e / o}=I_{f} \pm \frac{I_{b}-I_{f}}{e^{\beta\left(\Omega_{b}-\Omega_{f}\right)} \pm 1}
$$

where the upper/lower sign corresponds to the even/odd ensemble and we have defined

$$
I_{e / o}=-c\left(\frac{\partial \Omega_{e / o}}{\partial \Phi}\right)_{\mu(\Phi)}, \quad I_{f / b}=-c\left(\frac{\partial \Omega_{f / b}}{\partial \Phi}\right)_{\mu(\Phi)} .
$$

Below we will make use of these equations in order to evaluate $\mathrm{PC}$ in superconducting nanorings.

\section{Superconducting rings with nanojunctions}

\subsection{The model and general analysis}

Before we turn to concrete calculations let us specify the model for our system. In what follows we will investigate $\mathrm{PC}$ in superconducting nanorings with cross section $s$ and perimeter $L=2 \pi R$. Superconducting properties of such rings will be described within the (parity projected) mean field BCS theory. A necessary - though not always sufficient - validity condition for this mean field approach reads $N_{\mathrm{r}} \sim p_{F}^{2} s \gg 1$, i.e. the total number of conducting channels in the ring $N_{\mathrm{r}}$ should remain large. More detailed requirements can be formulated with the aid of the results $[10,11]$. For generic wires QPS effects are small provided the parameter $\sqrt{s}$ exceeds $\sim 10 \mathrm{~nm}[10,11,12,13]$. Considering then homogeneous rings one can prove [7] that in such rings the effect of the electron parity number on $\mathrm{PC}$ remains small in the parameter $\sim 1 / N_{\mathrm{r}} \ll 1$. On the other hand, in rings with only few number of channels $[8,9] N_{\mathrm{r}} \sim 1$ fluctuation effects may entirely wipe 


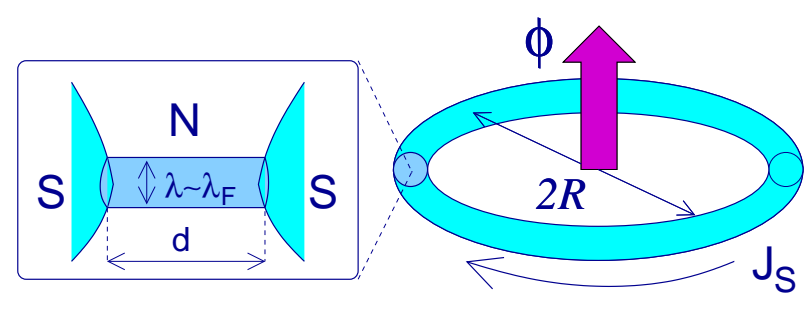

Fig. 1. Superconducting ring with embedded $S N S$ junction of length $d$.

out the supercurrent thus making the mean field approach obsolete.

The way out is to consider superconducting rings with $N_{\mathrm{r}} \gg 1$ interrupted by a weak link with only few number of conducting channels $N$. In such systems the mean field BCS description remains applicable and, on the other hand, the parity effect can be large due to the condition $N \sim 1$. Quantum point contacts (QPC) or, more generally, $S N S$ junctions (of length $d$ ) can be used for practical realization of such weak links. The corresponding structure is depicted in Fig. 1.

In this case the thermodynamic potential of the system $\Omega$ consists of two different contributions [14]

$$
\Omega=\Omega^{(r)}\left(\mu, T, \Phi_{x}, \varphi\right)+\Omega^{(c)}(\mu, T, \varphi)
$$

respectively from the bulk part of the ring and from the contact. The optimal value of the phase difference $\varphi$ across the weak link is fixed by the condition $\partial \Omega / \partial \varphi=$ 0 which reads

$$
-c \frac{\partial \Omega^{(r)}}{\partial \Phi_{x}}=-\frac{2 e}{\hbar} \frac{\partial \Omega^{(c)}}{\partial \varphi} .
$$

In (8) we made use of the fact that the thermodynamic potentials of the ring depend both on $\Phi_{x}$ and $\varphi$ only via the superfluid velocity $v_{S}=(1 / 4 \pi m R)\left(\varphi-2 \pi \Phi_{x} / \Phi_{0}\right)$, in which case one can put $\partial / \partial \Phi_{x}=-(2 e / \hbar c)(\partial / \partial \varphi)$. Here and below $\Phi_{0}$ is the superconducting flux quantum. The left-hand side of Eq. (8) represents the current flowing inside the superconducting $\operatorname{ring} I^{(r)}=$ $-c \partial \Omega^{(r)} \partial \Phi_{x} \simeq\left(e v_{F} N_{\mathrm{r}} / L\right)\left(\varphi-2 \pi \Phi_{x} / \Phi_{0}\right)$. This value should be equal to the current across the weak link which is given by the right-hand side of Eq. (8). Estimating the maximum value of the latter for a single channel contact as $2 e \mathcal{T} \Delta / \hbar$, we obtain

$$
\begin{array}{lll}
\varphi \simeq 2 \pi \frac{\Phi_{x}}{\Phi_{0}}, & \text { if } & L \ll L^{*}, \\
\varphi \simeq 2 \pi n, & \text { if } & L \gg L^{*},
\end{array}
$$

where $L^{*}=\xi_{0} N_{\mathrm{r}} / \mathcal{T} \gg \xi_{0}$. In a more general case of weak links with $N$ conducting channels in the expression for $L^{*}$ one should set

$$
\mathcal{T} \rightarrow \sum_{n}^{N} \mathcal{T}_{n}
$$

In what follows we will consider the most interesting limit $N \ll N_{\mathrm{r}}$ and $L \ll L^{*}$. Due to Eq. (9) in this case the dependence $I_{e / o}\left(\Phi_{x}\right)$ is fully determined by the current-phase relation for QPS which can be found by means of Eq. (6) with $I_{f / b}=-(2 e / \hbar) \partial \Omega_{f / b}^{(c)} / \partial \varphi$. We also note that quantum fluctuations of $\varphi$ can easily be suppressed, e.g. by choosing the ring inductance sufficiently small. In accordance with the charge-phase uncertainty relation the number of Cooper pairs passing through the junction should strongly fluctuate thereby suppressing charging effects in the contact area.

Let us now directly evaluate the supercurrent in both even and odd ensembles. As it was demonstrated above, it is sufficient to find the currents $I_{f / b}$ along with the difference of thermodynamic potentials $\Omega_{b}-\Omega_{f} \equiv$ $\Omega_{b f}$ and combine the results with Eq. (6). The value $I_{f / b}$ can be determined by means of a general formula [15]

$$
I_{f / b}=\frac{2 e}{\hbar} \sum_{i=1}^{N} T \sum_{\omega_{f / b}} \frac{\sin \varphi}{\cos \varphi+W_{i}\left(\omega_{f / b}\right)},
$$

and $\Omega_{b f}$ is evaluated as a sum of the ring and the weak link contributions, $\Omega_{b f}=\Omega_{b f}^{(r)}+\Omega_{b f}^{(w l)}$. The term $\Omega_{b f}^{(w l)}$ is found by integrating $I_{f / b}(\varphi)$ over the phase $\varphi$, while the ring contribution $\Omega_{b f}^{(r)}$ at low enough $T$ is defined by the standard expression $[2,4,5] \Omega_{b f}^{(r)} \simeq$ $\nu \mathcal{V} \Delta^{1 / 2} T^{3 / 2} \exp (-\Delta / T)$, where $\nu$ is the density of states at the Fermi level, $\mathcal{V}=s L$ is the ring volume and $\Delta$ is the superconducting energy gap.

\subsection{Rings with quantum point contacts}

Let us first consider the limit of short QPC with $d \rightarrow$ 0 . In this case one has $W_{i}(\omega)=\left(2 / \mathcal{T}_{i}\right)\left(1+\omega^{2} / \Delta^{2}\right)-$ 1 , where $\mathcal{T}_{i}$ is the transmission of the $i$-th channel. Combining this formula with Eq. (11), after a simple calculation from Eq. (6) one finds [7]

$$
I_{e / o}=-\frac{2 e}{\hbar} \sum_{i=1}^{N} \frac{\partial \varepsilon_{i}(\varphi)}{\partial \varphi} \tanh \frac{\varepsilon_{i}(\varphi)}{2 T}
$$




$$
\times\left[1 \pm \frac{\left(\operatorname{coth} \frac{\varepsilon_{i}(\varphi)}{2 T}\right)^{2}-1}{e^{\beta \Omega_{b f}^{(r)}} \prod_{j=1}^{N}\left(\operatorname{coth} \frac{\varepsilon_{j}(\varphi)}{2 T}\right)^{2} \pm 1}\right]
$$

where $\varepsilon_{i}(\varphi)=\Delta \sqrt{1-\mathcal{T}_{i} \sin ^{2}(\varphi / 2)}$. The term in the square brackets is specific for canonical ensembles and should be substituted by one for grand canonical ones. For $N=1$ and at $T=0$ this term reduces to unity for even ensembles and to zero for odd ones. In other words, in the latter case PC will be completely blocked by the odd electron and no current will flow in the system despite the presence of a non-zero external flux threading the ring. In QPC with several conducting channels the current through the most transparent one will be blocked by the odd electron and, hence, the effect can remain significant also for QPC with $N>1$.

The physics of this blocking effect was discussed in details in Ref. 7. It is related to a complete cancellation of the contributions to the supercurrent coming from a pair of discrete Andreev levels with energies $E_{ \pm}(\varphi)=$ $\pm \varepsilon_{i}(\varphi)$. Indeed, for the even ensemble at $T=0$ only the lower Andreev level $E_{-}(\varphi)$ is occupied, while the upper one $E_{-}(\varphi)$ remains empty. Accordingly, only the lower Andreev level contributes to the supercurrent in this case, and the result coincides with the standard grand canonical expression $[16,17,18,19]$. In contrast, for the odd ensemble both levels $E_{ \pm}(\varphi)$ are occupied at $T=0$ and contribute to the supercurrent in equal measure. These contributions, however, enter with opposite signs and completely cancel each other thus reducing the net supercurrent down to zero. We also note that a similar result was recently obtained in Ref. [20]

It is important to emphasize that the above physical picture is based on the assumption that the supercurrent is determined only by the contributions of two discrete Andreev levels $E_{ \pm}(\varphi)$. This is the case provided (a) quasiparticle states with energies above $\Delta$ do not contribute to the supercurrent and (b) there are no more discrete Andreev levels inside the junction. Both these conditions are met only for symmetric and extremely short QPC with $d \rightarrow 0$. In a general case $d \neq 0$ at least one of these conditions is violated, and no exact compensation of the supercurrent by the odd electron countercurrent is anymore possible. The physics then becomes even more interesting, as it will be demonstrated below.

\subsection{Rings with transparent $S N S$ junctions}

From now on let us lift the condition $d \rightarrow 0$, i.e. consider $S N S$ junctions with a non-zero thickness of the normal metal. Since the parity effect becomes more pronounced with increasing transmission of the system, let us first restrict our analysis to fully transparent $S N S$ junctions. In this case the function $W_{i}(\omega) \equiv$ $W(\omega)$ is the same for all channels. It reads

$$
\begin{gathered}
W(\omega)=\left(\frac{2 \omega^{2}}{\Delta^{2}}+1\right) \cosh \left(\frac{2 \omega d}{\hbar v_{F}}\right) \\
+\frac{2 \omega}{\Delta} \sqrt{1+\frac{\omega^{2}}{\Delta^{2}}} \sinh \left(\frac{2 \omega d}{\hbar v_{F}}\right) .
\end{gathered}
$$

Substituting this function into (11) and repeating the the whole calculation as above, in the limit $T \rightarrow 0$ we obtain

$I_{e}=\frac{e \Delta N}{\hbar}\left(\sin \frac{\varphi}{2}-\frac{2 y \sin \varphi}{\pi} \ln \frac{1}{y}\right)$,
$I_{o}=I_{e}-\frac{e \Delta}{\hbar}\left(\sin \frac{\varphi}{2}+y \operatorname{sgn} \varphi \cos \varphi\right)$

for short $S N S$ junctions $y \equiv d \Delta / \hbar v_{F} \ll 1$ and

$$
I_{e}=\frac{e v_{F} N}{\pi d} \varphi, \quad I_{o}=\frac{e v_{F} N}{\pi d}\left(\varphi-\frac{\pi \operatorname{sgn} \varphi}{N}\right)
$$

for long ones $d \gg \xi_{0} \sim \hbar v_{F} / \Delta$. These results apply for $-\pi<\varphi<\pi$ and should be $2 \pi$-periodically continued otherwise. The term containing $\ln (1 / y)$ in Eq. (15) for $I_{e}$ is written with the logarithmic accuracy and is valid for $\varphi$ not too close to $\varphi= \pm \pi$.

At $T=0$ the current $I_{e}$ again coincides with that for the grand canonical ensembles $[21,22]$. At the same time for odd ensembles we observe no blocking effect anymore, but rather a non-zero current $I_{o}$ for any nonzero value of $d$, cf. Eqs. (15-16). As before, at $T=0$ the countercurrent produced by the odd electron exactly compensates the current of the next lower Andreev level. However, the contribution of continuous spectrum (in the case $d \ll \xi_{0}$ ) or of continuous spectrum and all other discrete Andreev levels (in the case $\left.d \gg \xi_{0}\right)$ remains uncompensated and is responsible for the non-zero current $I_{o}$ (15-16).

It is important to emphasize that such a current causes a jump on the current-phase dependence at $\varphi=$ 0 , and the direction of $I_{o}$ at sufficiently small $\varphi$ is always opposite to that of $I_{e}$. This property implies that in the case of odd ensembles the minimum energy (zero 
current) state occurs not at $\varphi=0$, but at some other value of the phase difference. For instance, for $d \gg \xi_{0}$ the "saw tooth" current-phase relation is shifted by the value $\pi / N$ and, hence, the minimum Josephson energy (zero current) state is reached at $\varphi= \pm \pi / N$. One concludes that the " $\pi / N$-junction" state is realized in this case. This non-trivial state has a number of specific features. For instance, in the particular case $N=2$ the current-phase relation $I_{o}(\varphi)$ turns $\pi$-periodic. In addition, for any $N>1$ the ground state of the system $\varphi=$ $\pm \pi / N$ is a twofold degenerate one within the interval $-\pi<\varphi<\pi$. Here we note that a similar behavior is expected for $S N S$ junctions formed by $d$-wave superconductors [24].

Let us also recall that the $\pi$-junction state can be realized in $S N S$ structures by driving the electron distribution function in the contact area out of equilibrium $[25,26,27]$. Here, in contrast, the situation of a $\pi$ or $\pi / N$-junction is achieved in thermodynamic equilibrium. Despite this drastic difference, there also exists a certain physical similarity between the effects discussed here and in Refs. [25,26,27]: In both cases the electron distribution function in the weak link deviates substantially from the Fermi function. It is this deviation which is responsible for the appearance of the $\pi$-junction state in both physical situations.

The current-phase relations $I_{e}(\varphi)$ and $I_{o}(\varphi)$ for arbitrary values of the parameter $y$ can be computed numerically. Examples are presented in Fig. 2. In the case of odd ensembles one clearly observes the current jump at $\varphi=0$. As it is obvious from Eq. (15), this feature disappears only in the QPC limit $y \rightarrow 0$.

\subsection{Spontaneous currents in the ground state}

Perhaps the most interesting property of superconducting rings interrupted by a $\pi$-junction is the possibility to develop spontaneous supercurrent in the ground state [23]. Although this feature is inherent to any type of $\pi$-junctions, in the case of the standard sinusoidal current-phase relation such spontaneous supercurrents can occur only for sufficiently large values of the ring inductance $\mathcal{L}$ [23]. In contrast, in the situation studied here the spontaneous current state is realized for any inductance of the ring because of the non-sinusoidal dependence $I_{o}(\varphi)(16)[28]$.

In order to demonstrate that let us assume that no

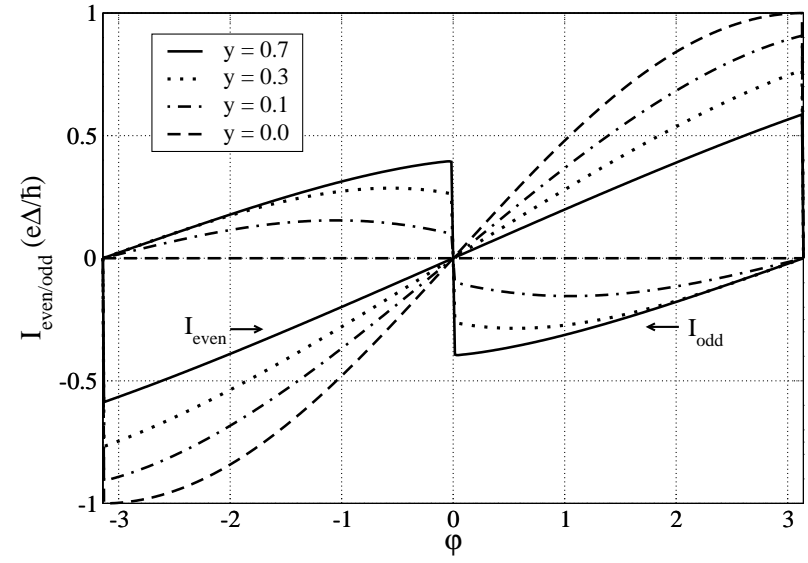

Fig. 2. The zero temperature current-phase relations $I_{e}(\varphi)$ and $I_{o}(\varphi)(-\pi<\varphi<\pi)$ for $N=1$ and different values of the parameter $y=d \Delta / \hbar v_{F}$.

external flux is applied to our system. Then at $T \rightarrow 0$ the energy of an $S N S$ ring with odd number of electrons can be written in the form

$$
E_{o}=\frac{\Phi^{2}}{2 c \mathcal{L}}+\frac{\pi \hbar v_{F} N}{\Phi_{0}^{2} d}\left(\Phi-\frac{\Phi_{0} \operatorname{sgn} \Phi}{2 N}\right)^{2},
$$

where $\Phi$ is the flux related to the circular current flowing in the ring. Minimizing this energy with respect to $\Phi$, one easily observes that a non-zero spontaneous current

$$
I= \pm \frac{e v_{F}}{d}\left[1+\frac{2 e v_{F} N}{d} \frac{\mathcal{L}}{\Phi_{0}}\right]^{-1}
$$

should flow in the ground state of our system. This is yet one more remarkable consequence of the parity effect: Just by changing $\mathcal{N}$ from even to odd one can induce non-zero PC without any external flux $\Phi_{x}$. In the limit of small inductances $\mathcal{L} \ll \Phi_{0} d / e v_{F} N$ - which is easy to reach in the systems under consideration - the value of $I$ does not depend on the number of channels $N$ and is given by the universal expressions can easily be derived from Eqs. (15-16):

$$
\begin{gathered}
I_{s p}=e \Delta^{2} d / \hbar^{2} v_{F}, \quad \text { if } d \ll \xi_{0}, \\
I_{s p}=e v_{F} / \pi d, \quad \text { if } d \gg \xi_{0} .
\end{gathered}
$$

For intermediate values of the parameter $y$ the amplitude of the current $I_{s p}$ can be evaluated numerically. The results are displayed in Fig. 3. In agreement with Eq. (19) the spontaneous current $I_{s p}$ increases linearly with $d$ at small $d$, reaches its maximum value $I_{\max } \sim$ $0.4 e \Delta / \hbar$ at $d \sim \xi_{0}$ and then decreases with further 


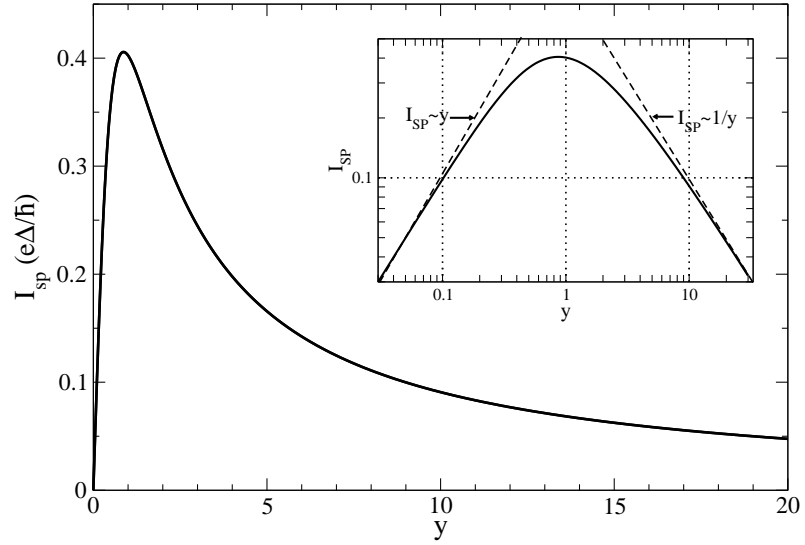

Fig. 3. The spontaneous current amplitude $I_{s p}$ as a function of the parameter $y=d \Delta / \hbar v_{F}$ at $T=0$. In the inset, the same function is shown on the $\log -\log$ scale. Dashed lines indicate the asymptotic behavior of $I_{s p}(y)$ in the limits of small and large $y$.

increase of $d$ approaching the dependence (20) in the limit of large $d$. For typical BCS superconductors this maximum current can be estimated as $I_{\max } \sim 10 \div 100$ $\mathrm{nA}$. Spontaneous currents of such a large magnitude can be directly detected in modern experiments.

\subsection{Junctions with resonant transmission}

As we have already emphasized above, the effects discussed here are mostly pronounced for $S N S$ junctions with few conducting channels $N \sim 1$. The most promising candidates for practical realization of such structures are junctions with $N$-layers formed by carbon nanotubes or organic molecules. In both these cases it is not always easy to achieve good quality contacts between nanotubes/molecules and superconducting electrodes. In other words, although the electron transport in the $N$-metal itself can be ballistic, electron scattering at $N S$-interfaces may be substantial and may significantly modify the above results.

At the first glance, in the limit of small transmission of $N S$-interfaces the influence of the parity effect on PC should become weak and, hence, can be disregarded. Below we will demonstrate that this conclusion - although correct under certain conditions - does not necessarily apply to nanojunctions for which size quantization of energy levels is an important effect. This effect gives rize to resonant transmission of elec- trons across the junction and considerably modifies the physical situation.

To proceed we will adopt the standard model of an SINIS junction ("I" stands for the insulating layer) and assume that transmissions of both $N S$ interfaces are much smaller than unity. In this limit electron transport across the junction is mainly due to resonant tunneling through discrete energy levels inside the normal metal. For simplicity we will restrict our analysis to a single channel junction $N=1$. If needed, generalization of our analysis to the case $N>1$ can be performed in a straightforward manner.

As before, we will make use of the general expression (11) where the function $W(\omega)$ now takes the form [15]

$$
\begin{array}{r}
W(\omega)=\frac{4 \sqrt{\mathcal{R}_{1} \mathcal{R}_{2}}}{\mathcal{T}_{1} \mathcal{T}_{2}} \frac{\Omega^{2}}{\Delta^{2}} \cos \left(2 k_{F} d+\phi\right) \\
+\frac{\Omega^{2}\left(1+\mathcal{R}_{1}\right)\left(1+\mathcal{R}_{2}\right)+\omega^{2} \mathcal{T}_{1} \mathcal{T}_{2}}{\mathcal{T}_{1} \mathcal{T}_{2} \Delta^{2}} \cosh \left(\frac{2 \omega d}{\hbar v_{F}}\right) \\
+\frac{2\left(1-\mathcal{R}_{1} \mathcal{R}_{2}\right)}{\mathcal{T}_{1} \mathcal{T}_{2}} \frac{\Omega \omega}{\Delta^{2}} \sinh \left(\frac{2 \omega d}{\hbar v_{F}}\right) .
\end{array}
$$

Here $\mathcal{T}_{1,2}$ and $\mathcal{R}_{1,2}=1-\mathcal{T}_{1,2}$ are respectively transmission and reflection coefficients of the two $N S$ interfaces, $\Omega=\sqrt{\Delta^{2}+\omega^{2}}$ and $2 k_{F} d+\phi$ is the phase accumulated by electrons during the motion between the barriers.

In accordance with our assumption below we shall consider the limit $\mathcal{T}_{1,2} \ll 1$. The most interesting physical situation is realized in the limit of short SINIS junction $d \ll \xi_{0} \sim \hbar v_{F} / \Delta$. Under the above conditions the size energy level quantization in the normal metal becomes an important effect. In the case of a one dimensional metal of length $d$, the level spacing in the vicinity of the Fermi energy is $\delta \epsilon \sim \hbar v_{F} / d$. Hence, the condition for the short junction regime can also be represented in the form $\delta \epsilon \gg \Delta$. Tunneling of electrons into the reservoirs causes a non-zero linewidth of the energy levels which is proportional to $\mathcal{T}_{1,2} \delta \epsilon$. This value is much smaller than $\delta \epsilon$, hence, the resonances remain well separated. In this situation it suffices to take into account only the closest to the Fermi energy level inside the normal metal.

Let us introduce the energy of the resonant level $\epsilon_{R}$ by means of the relation $\cos \left(2 k_{F}+\phi\right) \approx-1+1 / 2\left(\frac{\epsilon_{R}}{\delta \epsilon}\right)^{2}$, where $\delta \epsilon=\hbar v_{F} / 2 d$, and the tunneling rates $\Gamma_{1,2} / \hbar=$ $\mathcal{T}_{1,2} \delta \epsilon / \hbar$. Expanding the cosh and sinh terms in Eq. (21) to the leading order in $\Delta / \delta \epsilon$ and substituting the result in Eq. (11), we obtain 
a

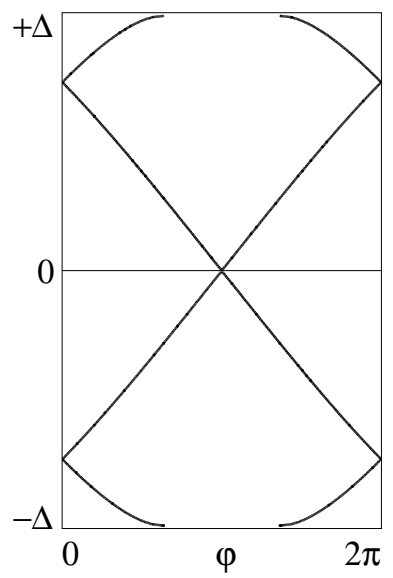

b

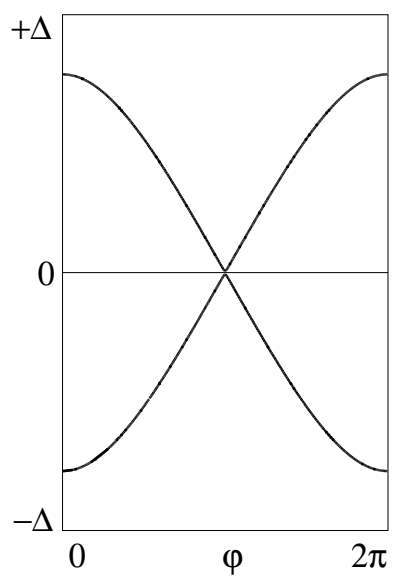

Fig. 4. Andreev levels in a single mode $S N S$ junction with $d=\hbar v_{F} / \Delta$ : (a) $\mathcal{T}_{1,2}=1$ and (b) $\mathcal{T}_{1,2} \ll 1$ and $\Delta / \Gamma=0.5$. In both cases $\mathcal{T}=1$.

$$
I_{f / b}=\frac{e}{\hbar} T \sum_{\omega_{f / b}} \frac{\Delta^{2} \mathcal{T} \sin \varphi}{\varepsilon^{2}(\varphi)+\omega_{f / b}^{2}\left(1+4 \mathcal{D} \mathcal{T} / \mathcal{T}_{\max }\right)},
$$

where

$$
\mathcal{D}=\left(\frac{\Delta}{\Gamma}\right)^{2}\left(1+\frac{\omega_{f / b}^{2}}{\Delta^{2}}\right)+\frac{\Delta}{\Gamma} \sqrt{1+\frac{\omega_{f / b}^{2}}{\Delta^{2}}},
$$

$\varepsilon(\varphi)=\Delta \sqrt{1-\mathcal{T} \sin ^{2}(\varphi / 2)}, \Gamma=\Gamma_{1}+\Gamma_{2}, \mathcal{T}_{\max }=$ $4 \Gamma_{1} \Gamma_{2} / \Gamma^{2}$ and the total transmission probability at the Fermi energy $\mathcal{T}$ is given by the Breit-Wigner formula

$$
\mathcal{T}=\frac{\Gamma_{1} \Gamma_{2}}{\left(\epsilon_{R}\right)^{2}+\frac{1}{4} \Gamma^{2}}
$$

It is interesting to point out that the expressions defined by Eqs. $(22,23)$ can also be derived from the Anderson model for resonant tunneling through a single impurity center between two superconductors in the limit of zero on-site interaction.

It follows from Eqs. $(22,23)$ that - although the transparencies of both barriers are low - the total transmission $\mathcal{T}$ and, hence, the Josephson current show sharp peaks provided the Fermi energy becomes close to the bound states inside the junction. On the other hand, Eqs. $(22,23)$ demonstrate that even in the vicinity of resonances the behavior of the Josephson current as a function of the phase difference $\varphi$ and temperature $T$ can substantially deviate from that for transparent $S N S$ junctions.

In order to understand the physical reasons for such a difference it is instructive to compare the structure

of discrete Andreev levels for ballistic $\left(\mathcal{T}_{1,2}=1\right) S N S$ junctions with that for $S I N I S$ junctions with $\mathcal{T}_{1,2} \ll$ 1, see Fig. 4. The spectrum of an SINIS system consists only of a single non-degenerate state $\varepsilon_{0}(\varphi)$ in the interval $0<\varepsilon_{0}<\Delta$ (Fig. 4b). As a result, the behavior of $\varepsilon_{0}(\varphi)$ at small $\varphi$ is smooth and the derivative of $\varepsilon_{0}$ with respect to $\varphi$ has no jump at $\varphi=0$. In contrast, in the case of ballistic $S N S$ junctions discrete levels become split at arbitrary small values of the phase $\varphi$ (Fig. 4a). Hence, the derivative of the lowest Andreev level with respect to $\varphi$ acquires a jump at $\varphi=0$. As we have already discussed, this feature is crucial for the presence of spontaneous currents in superconducting rings with arbitrary small inductances $\mathcal{L}$ and with odd number of electrons. Since this feature is absent in the case of SINIS junctions the spontaneous current in the ground state of the system can only develop at not very small ring inductances.

Analytical expressions for the energy of the subgap state (obtained from the poles of the summand in Eq. (22)) and for the parity-dependent currents $I_{e / o}$ can be derived in various asymptotic regimes. At a given value of the total transmission $\mathcal{T}$ one can distinguish two limiting cases: $\Delta / \Gamma \ll 1$ and $\Delta / \Gamma \gg 1$. In the case of a wide resonance $\Delta / \Gamma \ll 1$, the energy of the subgap state is $\varepsilon_{0}(\varphi)=\Delta \sqrt{1-\mathcal{T} \sin ^{2}(\varphi / 2)}$ and $I_{e / o}$ are given by the expressions derived for a single mode QPC with an effective transmission $\mathcal{T}$. In the opposite narrow resonance limit $\Delta / \Gamma \gg 1$ we get

$$
\varepsilon_{0}(\varphi)=\sqrt{\left(\epsilon_{R}\right)^{2}+\frac{1}{4} \Gamma^{2}} \sqrt{1-\mathcal{T} \sin ^{2}(\varphi / 2)} .
$$

The currents $I_{e / o}$ are again given by the expressions obtained for QPC with the substitution of $\Delta$ by $\sqrt{\left(\epsilon_{R}\right)^{2}+\frac{1}{4} \Gamma^{2}}$. Note that in both these asymptotic regimes only the discrete spectrum contributes to the currents $I_{e / o}$. Thus, for these regimes the Josephson current will be completely blocked by the odd electron in the limit of low temperatures.

At intermediate values of the parameter $\Delta / \Gamma$ the continuous spectrum will contribute to the Josephson current. The current-phase relations $I_{e / o}(\varphi)$ can be easily evaluated numerically for arbitrary parameter values. The results of these calculations - partially represented in Fig. 5 - clearly demonstrate that at sufficiently low temperatures the " $\pi$-junction" state should be realized in the case of odd number of electrons. According to our expectations, however, in the case of 


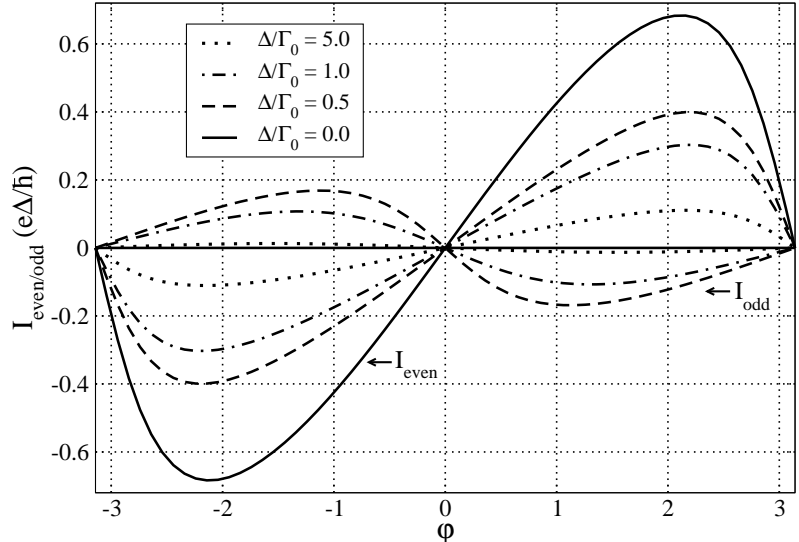

Fig. 5. Zero temperature current-phase relations $I_{e / o}(\varphi)$ for $\mathcal{T}=0.9, \Gamma_{1}=\Gamma_{2}=\Gamma_{0}$ and different values of the parameter $\Delta / \Gamma_{0}$.

resonant SINIS junctions the current has no jump at $\varphi=0$ and the behavior of $I_{o}(\varphi)$ is more similar to that of "conventional" $\pi$-junctions [23].

Let us emphasize again that all the above results for SINIS junctions with small interface transmissions were derived in the limit of short junctions $d \ll \xi_{0}$. Resonant effects are also important in the opposite limit $d \gg \xi_{0}[15]$. However, in the latter case even exactly at resonance the Josephson current remains small being proportional to the smallest of the two transmissions $\mathcal{T}_{1}$ and $\mathcal{T}_{2}$. Hence, the parity effect in the limit $d \gg \xi_{0}$ will also be small in equal measure. For this reason the case of long junctions with resonant transmission will not be considered here.

\section{Conclusions}

In this paper we have demonstrated that at sufficiently low temperatures superconducting parity effect may strongly influence equilibrium persistent currents in isolated superconducting nanorings containing a weak link with few conducting modes. An odd electron, being added to the ring, occupies the lowest available Andreev state and produces a countercurrent circulating inside the ring. For a single channel quantum point contact at $T=0$ this countercurrent exactly compensates the supercurrent $I_{e}$ produced by all other electrons and, hence, yields complete blocking of PC for any value of the external magnetic flux. In supercon- ducting nanorings with embedded normal metal the odd electron countercurrent can "overcompensate" $I_{e}$ and a novel " $\pi / N$-junction" state occurs in the system. Changing the electron parity number from even to odd results in spontaneous supercurrent in the ground state of such rings without any externally applied magnetic flux. This and other novel effects predicted here can be directly tested in modern experiments. They can also be used for practical realization of superconducting qubits.

Acknowledgments This work is part of the Kompetenznetz "Funktionelle Nanostructuren" supported by the Landestiftung Baden-Württemberg gGmbH. This work has also been supported by the European Community's Framework Programme NMP4-CT2003-505457 ULTRA-1D "Experimental and theoretical investigation of electron transport in ultra-narrow 1-dimensional nanostructures".

\section{References}

[1] D.V. Averin and Yu.V. Nazarov, Phys. Rev. Lett. 69 (1992) 1993.

[2] M.T. Tuominen, J.M. Hergenrother, T.S. Tighe, and M. Tinkham, Phys. Rev. Lett. 69 (1992) 1997.

[3] P. Lafarge, P. Joyez, D. Esteve, C. Urbina, and M.H. Devoret, Phys. Rev. Lett. 70 (1993) 994.

[4] B. Janko, A. Smith, and V. Ambegaokar, Phys. Rev. B 50 (1994) 1152.

[5] D.S. Golubev and A.D. Zaikin, Phys. Lett. A195 (1994) 380.

[6] D.V. Averin and Yu.V. Nazarov, Physica B203 (1994) 310.

[7] S.V. Sharov and A.D. Zaikin, Phys. Rev. B 71 (2005) 014518.

[8] K. Kang, Europhys. Lett. 51 (2000) 181.

[9] H.-J. Kwon and V.M. Yakovenko, Phys. Rev. Lett. 89 (2002) 017002.

[10] A.D. Zaikin, D.S. Golubev, A. van Otterlo, and G.T. Zimányi, Phys. Rev. Lett. 78 (1997) 1552.

[11] D.S. Golubev and A.D. Zaikin, Phys. Rev. B 64 (2001) 014504 . 
[12] A. Bezryadin, C.N. Lau, and M. Tinkham, Nature (London) 404 (2000) 971.

[13] C.N. Lau, N. Markovic, M. Bockrath, A. Bezryadin, and M. Tinkham, Phys. Rev. Lett. 87 (2001) 217003.

[14] The relation (7) is strictly applicable only for grand canonical ensembles. However, at this point the difference between canonical and grand canonical ensembles is unimportant and can be disregarded.

[15] A.V. Galaktionov and A.D. Zaikin, Phys. Rev. B 65 (2002) 184507.

[16] I.O. Kulik and A.N. Omel'yanchuk, Sov. J. Low Temp. Phys. 4 (1978) 142.

[17] W. Haberkorn, H. Knauer, and J. Richter, Phys. Stat. Solidi (A) 47 (1978) K161.

[18] A. Furusaki and M. Tsukada, Physica B165-166 (1990) 967.

[19] C.W.J. Beenakker and H. van Houten, Phys. Rev. Lett. 66 (1991) 3056.

[20] M. Hayashi and H. Ebisawa, Phys. Rev. B 67 (2003) 014524.

[21] I.O. Kulik, Sov. Phys. JETP 30 (1970) 944.

[22] C. Ishii, Progr. Theor. Phys. 44 (1970) 1525.

[23] L.N. Bulaevskii, V.V. Kuzii, and A.A. Sobyanin, JETP Lett. 25 (1977) 290.

[24] Yu.S. Barash, A.V. Galaktionov and A.D. Zaikin, Phys. Rev. B 52 (1995) 665.

[25] A.F. Volkov, Phys. Rev. Lett. 74 (1995) 4730.

[26] F.K. Wilhelm, G. Schön, and A.D. Zaikin, Phys. Rev. Lett. 81 (1998) 1682.

[27] S.K. Yip, Phys. Rev. 58 (1998) 5803.

[28] For small $\mathcal{L} \rightarrow 0$ non-vanishing spontaneous currents in the ground state may as well exist in systems containing $S N S$ junctions formed by $d$-wave superconductors [24]. Also in that case this feature is due to the non-sinusoidal current-phase relation in such junctions. 